\title{
A generalisation of Turán's main theorems to binomials and logarithms
}

\author{
A. J. van der Poorten
}

\begin{abstract}
The Main Theorems of P. Turân's book Eine neue Methode in der Analysis und deren Anwendungen concern only sums of powers but are easily generalised to exponential sums with polynomial coefficients. It does not appear to have been observed however, that similar such theorems with analogous implication as to value distribution and arithmetical behaviour can be formulated for a wider class of functions. We prove a result for functions of the form $\sum p_{k q}(z)(1-z)^{\alpha} k_{\log ^{q-1}(1-z)}$ subsuming identities which Mahler has shown to contain transcendence results on the exponential and logarithmic functions and diophantine results of the Thue-Siegel-Roth type.
\end{abstract}

1.

Theorems of the nature of those developed by Turán in his book [8] lead directly to results on the distribution of values of the relevant functions

$$
\sum_{k=1}^{m} \sum_{s=1}^{\rho(k)} a_{k s} z^{s-1} e^{w_{k} z}=\sum_{k=1}^{m} p_{k}(z) e^{w_{k} z},
$$

as observed by Dancs and Turán [2]; see also results of Mahler [6] and Gelfond [3; p. 140]. The techniques employed in this work do not however appear susceptible of generalisation, and the rather remarkable result of Dancs and Turán giving an upper bound for the number of zeros of functions

Received 24 November 1969. 
of the form (1) in any square in the complex plane, so that the bound is independent of the coefficients $a_{k s}$ and only loosely dependent on exponents $w_{k}$, appears to be specific to entire functions of the indicated form.

A new technique, in effect a sledge-hammer approach, involving evaluation of determinants and their cofactors, will however apply in any circumstance where the evaluations can be conveniently performed. Elsewhere we have obtained new results generalising Turán's so-called Main Theorems [9], and a result on the distribution of zeros of functions of the form (1) independent of the quantity $\min _{h \neq k}\left|w_{h}-w_{k}\right|,[10,11]$. In this paper we prove the following result.

THEOREM. Let $\rho(h, q), n(h), 1 \leq h \leq m, 1 \leq q \leq n(h)$, be non-negative integers and let $\sigma$ be the sum of the $\rho(h, q)$. Further let $\alpha_{1}, \alpha_{2}, \ldots, \alpha_{m}$ be complex numbers, and let $F$ denote a function of the form

$$
\begin{aligned}
F(z) & =\sum_{h=1}^{m} \sum_{q=1}^{n(h)} \sum_{s=1}^{\rho(h q)} a_{h q s} z^{s-1}(1-z)^{\alpha} h_{1 \circ g^{q-1}}(1-z) \\
& =\sum_{h=1}^{m} \sum_{q=1}^{n(h)} p_{h q}(z)(1-z)^{\alpha} h_{1 \circ g^{q-1}(1-z)}
\end{aligned}
$$

where $\log ^{q-1}(1-z)$ denotes $\{\log (1-z)\}^{q-1}$ and $a_{\text {hqs }}, 1 \leq h \leq m$, $1 \leq q \leq n(h), \quad 1 \leq s \leq \rho(h, q)$ are complex constants so that the $p_{h q}(z)$ are polynomials of degree at most $\rho(h, q)-1$.

Finally let

$$
\rho(h, 1) \geq \rho(h, 2) \geq \ldots \geq \rho(h, n(h)), \quad 1 \leq h \leq m
$$

and write

$$
A=\max _{1 \leq h \leq m ; 1 \leq q \leq n(h) ; 1 \leq t \leq \rho(h q)}\left|t-1+\alpha_{h}\right| ;
$$

then for any point $u \neq 1$ 


$$
\max _{1 \leq \mu \leq \sigma}\left|\frac{(\sigma-\mu) !}{(\sigma-1) !} F^{(\mu-1)}(0)\right| \geq|F(u)| \frac{\min \left\{|1-u|,|1-u|^{-1}\right\}^{A+1}}{(\sigma+A)^{\sigma}} .
$$

With the aid of the theorem it is easy to obtain an upper bound for the number of zeros of $F(z)$ in small circles about the origin, such a bound being independent of the coefficients $a_{\text {hqt }}$, and in depending on $A$ only loosely dependent on the exponents $\alpha_{h}$. Unfortunately the singularity at $z=1$ interferes with more general results. The reader is referred to [11] for details of a convenient method of proof.

Functions of the form of those of the Theorem are discussed by A. Baker [1] who showed that under the conditions of the theorem and the additional restriction that no two of $\alpha_{1}, \alpha_{2}, \ldots, \alpha_{m}$ differ by a rational integer either $F(z) \equiv 0$ or $F(z)$ vanishes at most $\sigma-1$ times at $z=0$. This result, which follows immediately from our explicit evaluation of the determinant $\Delta$ (10), generalises results of Mahler on the binomial [4] and logarithmic [5] functions.

In other respects our results are new, and except for the exponential case, do not appear to have been remarked upon before. The feature that our results do not collapse when two of $\alpha_{1}, \alpha_{2}, \ldots, \alpha_{m}$ differ by an integer arises as a special benefit of our method; our evaluations are actually easier because of extra symmetry. The reader should note the analogous situation described in our paper [10]. Our results investigate $F(z)$ only in a neighbourhood of the origin; there does not appear to be any reason why we should not obtain similar results in any neighbourhood in which $F(z)$ remains non-singular. It is an exercise, perhaps of annoying arithmetical complexity, to confirm this, and to obtain more general results on the distribution of zeros of $F(z)$.

A further feature of our method deserving comment is that we are not forced to estimate crudely until the final step when we provide a tidy result. In consequence one is able to read of precise results for special cases from the expressions appearing in our proof.

2.

We will require the following subsidiary result. 
MAIN LEMMA. Let $G$ be a function of the form

$$
G(z)=\sum_{k=1}^{n} b_{k} g_{k}(z), b_{1}, \ldots, b_{n} \text { complex constants }
$$

where $g_{1}, \ldots, g_{n}$ are functions analytic on some domain $D$ of the complex plane.

Further let $z_{1}, \ldots, z_{n}$ be points of $D$; let $s_{1}, s_{2}, \ldots, s_{n}$ be non-negative integers; and let $H\left(y_{1}, \ldots, y_{2}\right)$ be a form Linear in $y_{1}, \ldots, y_{2}, \quad(1 \leq Z \leq n)$, and $c_{1}, \ldots, c_{n}$ non-zero constants. Finally denote by $\Delta_{i j}, 1 \leq i, j \leq n$ the cofactor of $g_{j}\left(s_{i}\right)\left(z_{i}\right)$ in the determinant

$$
\Delta=\left|g_{j}^{\left(s_{i}\right)}\left(z_{i}\right)\right|_{1 \leq j, i \leq n}
$$

Then there is an integer $\mu$ such that $1 \leq \mu \leq n$ and

$$
\left|\frac{1}{c_{\mu}}{ }_{\mu}^{\left(s_{\mu}\right)}\left(z_{\mu}\right)\right| \geq \frac{\mid H\left(b_{j(1)}, \ldots, b_{j(2)}|| \Delta \mid\right.}{\sum_{i=1}^{n}\left|c_{i} H\left(\Delta_{i j(1)}, \ldots, \Delta_{i j(z)}\right)\right|}, \quad 1 \leq j(1)<\ldots<j(2) \leq n .
$$

Proof. Appropriately differentiating at $z_{1}, z_{2}, \ldots, z_{n}$ we obtain a system of $n$ linear equations in $b_{1}, \ldots, b_{n}$

$$
\sum_{k=1}^{n} b_{k} g^{\left(s_{i}\right)}\left(z_{i}\right)=G^{\left(s_{i}\right)}\left(z_{i}\right), .1 \leq i \leq n \text {, }
$$

which we may solve by Cramer's rule to obtain for $1 \leq k \leq n$

$$
b_{k} \Delta=\sum_{i=1}^{n} \Delta_{i k} G^{\left(s_{i}\right)}\left(z_{i}\right) \text {. }
$$

Thus

$$
H\left(b_{j(1)}, \ldots, b_{j(1)}\right) \Delta=\sum_{i=1}^{n} H\left(\Delta_{i j(1)}, \ldots, \Delta_{i j(2)}\right) G^{\left(s_{i}\right)}\left(z_{i}\right)
$$

whence 


$$
\begin{aligned}
\left|H\left(b_{j(1)}, \ldots, b_{j(l)}\right)\right| & |\Delta| \\
\leq & \sum_{i=1}^{n}\left|c_{i} H\left(\Delta_{i j(1)}, \ldots, \Delta_{i j(z)}\right)\right| \cdot \max _{1 \leq \mu \leq n}\left|\frac{1}{c_{\mu} G}\left(s_{\mu}\right)\left(z_{\mu}\right)\right|
\end{aligned}
$$

and the assertion follows. We note that the result remains meaningful though trivial even if the denominator on the right-hand side of the result should vanish, provided we then interpret the lower bound to be zero (this is the natural interpretation, in our examples, by continuity).

With the notation of the theorem, we apply the Main Lemma to the function

$$
F(z)=\sum_{k=1}^{m} \sum_{q=1}^{n(k)} \sum_{s=1}^{\rho(k)} a_{k q s} z^{s-1}(1-z)^{\alpha} k^{\alpha \circ g}{ }^{q-1}(1-z),
$$

that is, to the $\sigma$ functions $z^{s-1}(1-z)^{\alpha} k \log ^{q-1}(1-z)$. We will then be considering a $\sigma \times \sigma$ determinant

$$
\begin{aligned}
& \Delta=\left\{\left.\left\{\left(\frac{d}{d z}\right)^{\lambda-1} z^{t-1}(1-z)^{\alpha} h_{10 g} q-1(1-z)\right\}_{z=0}\right|_{h q t, \lambda},\right. \\
& \qquad 1 \leq h \leq m ; 1 \leq q \leq n(h) ; 1 \leq t \leq \rho(h q) ; 1 \leq \lambda \leq \sigma .
\end{aligned}
$$

Here $h, q, t$ index rows and $\lambda$ indexes columns; the above $h q t, \lambda$ index indicates that we display the hat, $\lambda$ element. We always denote the cofactor, i.e. the determinant of the minor together with the appropriate sign, of the hqt, $\lambda$ element by $\Delta_{\lambda, h q t}$.

With this notation, the particular case of the lemma we use will be, $u \neq 1$.

$\max _{1 \leq \mu \leq \sigma}\left|\frac{(\sigma-\mu) !}{(\sigma-1) !} F^{(\mu-1)}(0)\right|$

$$
\begin{aligned}
& \geq \frac{\left|\sum_{h=1}^{m} \sum_{q=1}^{n(h)} \rho \sum_{t=1}^{\rho(h q)} u^{t-1}(1-u)^{\alpha}{ }^{\alpha} \log ^{q-1}(1-u) a_{h q t}\right||\Delta|}{\sum_{\lambda=1}^{\sigma}\left|\frac{(\sigma-1) !}{(\sigma-\lambda) !} \sum_{h, q, t} u^{t-1}(1-u)^{\alpha}{ }_{\log ^{q-1}(1-u) \Delta}{ }_{\lambda, h q t}\right|} \\
& =|F(u)|\left\{\left.\sum_{\lambda=1}^{\sigma} \frac{(\sigma-1) !}{(\sigma-\lambda) !}\right|_{h, q, t} u^{t-1}(1-u)^{\alpha} h_{\log } q-1(1-u) \frac{\Delta_{\lambda, h q t}}{\Delta} \mid\right\}^{-1}
\end{aligned}
$$


and our problem becomes that of finding an upper bound for the bracketed factor.

3.

Before commencing our proof it is appropriate to establish some notational conventions beyond those indicated in the introduction. Thus we will not indicate the ranges of indices in a formula if these have been clearly indicated in previous expressions, since otherwise our formulae will certainly explode into incomprehensibility. We will write, for non-negative $n$

$$
z^{(n)}=z(z-1)(z-2) \ldots(z-n+1), z^{(0)}=1,
$$

and

$$
\frac{\delta}{\delta z} f(z)=f(z+1)-f(z)
$$

creating a useful notational analogy with the usual derivative. The reader should note aspects of the behaviour of what we will call the difference operator (3), namely

$$
\begin{gathered}
\frac{\delta}{\delta z^{z}}(n)=n z^{(n-1)}, \\
\frac{\delta}{\delta z} g(z) f(z)=\left\{\frac{\delta}{\delta z} f(z)\right\} g(z)+f(z+1) \frac{\delta}{\delta z} g(z),
\end{gathered}
$$

and the Leibniz formula

$$
\left(\frac{\delta}{\delta z}\right)^{n} g(z) f(z)=\sum_{\lambda=0}^{n}\left(\begin{array}{l}
\lambda \\
n
\end{array}\right)\left\{\left(\frac{\delta}{\delta z}\right)^{n-\lambda} f(z+\lambda)\right\}\left(\frac{\delta}{\delta z}\right)^{\lambda} g(z) \text {. }
$$

4.

In order to apply the Main Lemma we will be required to evaluate the $\sigma \times \sigma$ determinant

$$
\begin{aligned}
& \Delta=\left|\left\{\left(\frac{d}{d z}\right)^{\lambda-1} z^{t-1}(1-z)^{\alpha} h_{\log }^{q-1}(1-z)\right\}_{z=0}\right|_{h q t, \lambda}, \\
& 1 \leq h \leq m ; 1 \leq q \leq n(h) ; 1 \leq t \leq \rho(h q) ; 1 \leq \lambda \leq \sigma .
\end{aligned}
$$

With some manipulation we observe that 
$\left\{\left(\frac{d}{d z}\right)^{\lambda-1} z^{t-1}(1-z)^{\alpha} h_{\log ^{q-1}}(1-z)\right\}_{z=0}=(-1)^{\lambda-t} \frac{(\lambda-1) !}{(\lambda-t) !}(q-1) !$. (sum of $\alpha_{h}, \alpha_{h}-1, \ldots, \alpha_{h}-\lambda+t+1$ taken $\lambda-t-q+1$ at a time)

$$
\begin{aligned}
& =(-1)^{\lambda-t+q-1} \frac{(\lambda-1) !}{(\lambda-t) !}\left\{\left(\frac{d}{d z}\right)^{q-1}\left(\alpha_{h}-z\right)^{(\lambda-t)}\right\}_{z=0} \\
& =(-1)^{\lambda-t+q-1}\left(\frac{\delta}{\delta \alpha_{h}}\right)^{t-1}\left\{\left(\frac{d}{d z}\right)^{q-1}\left(\alpha_{h}-z\right)^{(\lambda-1)}\right\}_{z=0} .
\end{aligned}
$$

Thus introducing formal quantities $\alpha_{h q t}$, $\beta_{h q t}$ we obtain, defining the determinant $D$ by

$$
D=\left|\left(\alpha_{h q t}-\beta_{h q t}\right)^{(\lambda-1)}\right|_{h q t, \lambda},
$$

that

$$
\text { (5) } \pm \Delta=\lim _{\substack{\alpha_{h q t} \rightarrow \alpha_{h} \beta_{h q t} \rightarrow 0 \\ 1 \leq h \leq m ; 1 \leq q \leq n(h) ; 1 \leq t \leq \rho(h q)}}\left\{\left\{\prod_{h ; q, t}\left(\frac{\partial}{\partial \beta_{h q t}}\right)^{q-1}\left(\frac{\delta}{\delta \alpha h q t}\right)^{t-1}\right\}_{D}\right) \text {, }
$$

the precise sign, indicated by \pm , being recoverable from (4) above. In the same way it is immediate that we obtain the cofactors of $\Delta$ by, up to sign,

(6) $\Delta_{\lambda, k p s}=\lim _{\alpha_{h q t} \rightarrow \alpha_{h}} \lim _{h q t}\left(\left\{\prod_{\substack{h, q, t \\ h q t \neq k p s}}\left(\frac{\partial}{\partial \beta_{h q t}}\right)^{q-1}\left(\frac{\delta}{\delta \alpha_{h q t}}\right)^{t-1}\right\}_{\lambda, k p s}\right)$.

In particular a simple calculation shows that, up to sign,

$$
\begin{aligned}
& u^{s-1}(1-u)^{\alpha_{k}}{ }_{1 \circ g}^{p-1}(1-u) \Delta_{\lambda, k p s}
\end{aligned}
$$

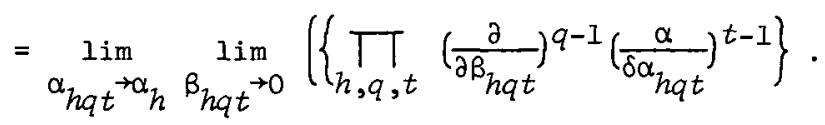

$$
\begin{aligned}
& \left.\cdot(-1)^{s+p}(1-u)^{\alpha p s}{ }^{-\beta_{k p s}}{ }_{\lambda, k p s}\right)
\end{aligned}
$$

since $D_{\lambda, k p s}$ has no terms in $\alpha_{k p s}, B_{k p s}$.

The object of our procedure is to obtain quantities which can be evaluated exactly. Since $D$ is in effect a Vandermonde alternant we immediately obtain 


$$
D=\prod_{k p s<h q t}\left\{\left(\alpha_{h q t}-\beta_{h q t}\right)-\left(\alpha_{k p s}-\beta_{k p s}\right)\right\}
$$

and under the assumption

$$
\rho(h l) \geq \rho(h 2) \geq \ldots \geq \rho(h n(h)), \quad 1 \leq h \leq m,
$$

after some reorganisation

$D=\prod_{h=1}^{m} \prod_{q=1}^{n(h)} \prod_{t=1}^{\rho(h q)}\left\{\prod_{s=1}^{t-1}\left\{\left(\alpha_{h q t}-\alpha_{h q s}\right)-\left(\beta_{h q t}-\beta_{h q s}\right)\right\}\right.$.

$$
\begin{aligned}
& \cdot \prod_{p=1}^{q-1}\left[\left\{\left(\alpha_{h q t}-\alpha_{h p t}\right)-\left(\beta_{h q t}-\beta_{h p t}\right)\right\}\right. \text {. } \\
& \text { - } \left.\prod_{\substack{l=1 \\
l \neq t}}^{p(h p)}\left\{\left(\alpha_{h p t}-\alpha_{h p l}\right)-\left(\beta_{h q t}-\beta_{h p l}\right)\right\}\right] \text {. } \\
& \left.\cdot \prod_{j=1}^{h-1} \prod_{r=1}^{n(j)} \prod_{i=1}^{\rho(j r)}\left\{\left(\alpha_{h q t}-\alpha_{j m i}\right)-\left(\beta_{h q t}-\beta_{j m i}\right)\right\}\right\} \text {. }
\end{aligned}
$$

In differentiating and differencing this product according to (5) we must eliminate the first two lines of terms, since otherwise on taking limits the resulting term in the sum we obtain will vanish. The reader should check that we differentiate and difference exactly so as to be able to eliminate these terms; compare the analogous step in [9] and [10]. In consequence we obtain exactly, up to sign,

$$
\begin{aligned}
& \Delta=\prod_{h, q, t}\left\{(t-1) !(q-1) !\left[\prod_{p=1}^{q-1}(\rho(h p)-t) !(t-1) !\right] .\right. \\
& \text { (10) } \left.\quad \cdot \prod_{j=1}^{n-1} \prod_{r=1}^{n(j)} \prod_{i=1}^{\rho(j r)}\left(\alpha_{h}-\alpha_{j}+t-i\right)\right\} .
\end{aligned}
$$

5.

Now consider the sum

$$
\sum_{k=1}^{m} \sum_{\rho=1}^{n(k)} \sum_{s=1}^{\rho(k p)}(1-u)^{\alpha p s s^{-\beta_{k p s}}} D_{\lambda, k p s},
$$

where the sign has been appropriately selected so that on applying our usual operator 
(12)

$$
\Omega=\operatorname{\alpha im}_{h q t} \lim _{h q \alpha_{h} \rightarrow 0}\left(\left\{\prod_{h, q, t}\left(\frac{\partial}{\partial \beta_{h q t}}\right)^{q-1}\left(\frac{\delta}{\delta \alpha_{h q t}}\right)^{t-1} \ldots\right\}\right)
$$

we obtain by (7) exactly, up to sign

$$
\sum_{k, p, s} u^{s-1}(1-u)^{\alpha} k_{\log ^{p-1}}(1-u) \Delta_{\lambda, k p s}
$$

But the sum of cofactors (11) is alternating in $\alpha_{h q t}-\beta_{h q t}$, all hqt and by well-known theorems on determinants is therefore divisible by the difference product $D$ as detailed in (9). We once again refer the reader to the paper [9; Lemma 3] for analogous details. Writing

$$
P=\prod_{h, q, t}\left\{\prod_{s=1}^{t-1}\left\{\left(\alpha_{h q t}-\alpha_{h q s}\right)-\left(\beta_{h q t}-\beta_{h q s}\right)\right\} \cdot \prod_{p=1}^{q-1}\left\{\left(\alpha_{h q t}-\alpha_{h p t}\right)-\left(\beta_{h q t}-\beta_{h p s}\right)\right\}\right\}
$$

one obtains

$\sum_{k, p, s} u^{s-1}(1-u)^{\alpha_{k}}{ }_{1 \circ g}^{p-1}(1-u) \frac{\Delta_{\lambda, k p s}}{\Delta}=\Omega\left\{\sum_{k, p, s}(1-u)^{\alpha_{k p s}-\beta_{k p s}} D_{\lambda, k p s}\right\} D$

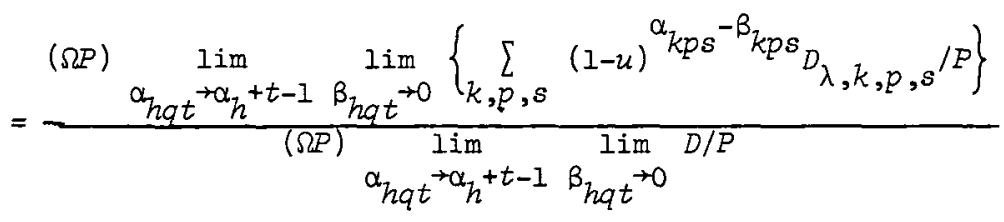

$$
\begin{aligned}
& =\lim _{h q t} \lim _{\alpha_{h}+t-1} \sum_{h q t}(1-u)^{\alpha_{k p s}-\beta_{k p s}} \frac{D_{\lambda, k p s}}{D}
\end{aligned}
$$

by virtue of the remarks we made when evaluating $\Delta$ from $D$, namely that other terms which appear by the action of $\Omega$ upon the indicated products vanish upon taking the appropriate limits.

6.

It is an elementary matter to evaluate the cofactors $D_{\lambda, k p s}$. We observe that the cofactors are defined by

$$
\sum_{\lambda=1}^{\sigma} D_{\lambda, k p s}\left(\alpha_{h q t}-\beta_{h q t}\right)^{(\lambda-1)}= \begin{cases}D & h q t=k p s, \\ 0 & \text { otherwise },\end{cases}
$$


whence $\frac{D_{\lambda, k p s}}{D}$ is exactly the coefficient of $z^{(\lambda-1)}$ in the polynomial $\prod_{h, q, t}\left(\frac{z-\alpha_{h q t}+\beta_{h q t}}{\left(\alpha_{k p s}-\alpha_{h q t}\right)-\left(\beta_{k p s}-\beta_{h q t}\right)}\right)$ expanded in the form $\sum_{\lambda=1}^{\sigma} a_{\lambda} z^{(\lambda-1)}$. hqt $\neq k p s$

Thus from (12) we obtain that

(14) $\sum_{k, p, s} u^{s-1}(1-u)^{\alpha k_{1 \circ g} p-1}(1-u) \frac{\Delta_{\lambda, k p s}}{\Delta}=$

$=$ the coefficient of $z^{(\lambda-1)}$ in the polynomial

$\lim _{\alpha_{h q t}+\alpha_{h}+t-1} \lim _{\beta_{h q t} \rightarrow 0} \sum_{k, p, s}(I-u)^{\alpha_{k p s}-\beta_{k p s}} \prod_{\substack{h, q, t \\ h q t \neq k p s}}\left(\frac{z-\alpha_{h q t}+\beta_{h q t}}{\left(\alpha_{k p s}-\alpha_{h q t}\right)-\left(\beta_{k p s}-\beta_{h q t}\right)}\right)$

$=$ the coefficient of $z^{(\lambda-1)}$ in the polynomial

(15)

$$
\begin{aligned}
\lim _{\alpha_{h q t}+\alpha_{h}+t-1} \sum_{k, p, s}(1-u)^{\alpha k p s} \prod_{\substack{h, q, t \\
h q t \neq k p s}}\left(\frac{z-\alpha_{h q t}}{\alpha_{k p s}-\alpha_{h q t}}\right) \\
=\text { the coefficient of } z^{(\lambda-1)} \text { in the polynomial }
\end{aligned}
$$

$$
\lim _{\alpha_{h q t}+\alpha_{h}} \sum_{k, p, s}(1-u)^{\alpha_{k p s}+s-1} \prod_{\substack{h, q, t \\ h q t \neq k p s}}\left(\frac{z-\alpha_{h q t}-t+1}{\alpha_{k p s}-\alpha_{h q t}+s-t}\right) .
$$

7.

Now consider the polynomial

$$
P(z)=\sum_{k, p, s}(1-u)^{\alpha_{k p s}+s-1} \prod_{\substack{h, q, t \\ h q t \neq k p s}}\left(\frac{z-\alpha_{h q t}-t+1}{\alpha_{k p s}-\alpha_{h q t}-t+s}\right) .
$$

$P(z)$ is defined by the $\sigma$ conditions

(18) $P\left(\alpha_{k p s}+s-1\right)=(1-u)^{\alpha_{k p s}+s-1}$,

$$
1 \leq k \leq m ; 1 \leq p \leq n(k) ; 1 \leq s \leq \rho(k p) .
$$

Thus expanding $P(z)$ as an interpolation series, 


$$
P(z)=\sum_{k, p, s} b_{k p s} \prod_{h q t<k p s}\left(z-\alpha_{h q t}-t+1\right),
$$

we can find the interpolation coefficients $b_{k p s}$ by, see for example, Nörlund's book $[7 ;$ p. 10],

$$
b_{k p s}=\frac{1}{2 \pi i} \int_{r} \frac{(1-u)^{\gamma} d \gamma}{f_{k p s}(\gamma)}
$$

where $f_{k p s}(\gamma)=\prod_{h q t \leq k p s}\left(\gamma-\alpha_{h q t}-t+1\right)$ and $\Gamma$ is the circumference $|\gamma|=R, R$ sufficiently large so that all poles of the integrand lie inside the contour. The integrals (20) permit us to find an upper bound for the interpolation coefficients; we will return to details subsequently.

The point of this approach is that the $b_{k p s}$ remain well defined even when we take the limits $\alpha_{h q t} \rightarrow \alpha_{h}$ so as to actually obtain the polynomial of (14) and its interpolation coefficients.

8.

In order to apply the Main Lemma we need an upper bound for

$$
S=\sum_{\lambda=1}^{\sigma}\left|a_{\lambda}\right| \frac{(\sigma-1) !}{(\sigma-\lambda) !}
$$

where $P(z)=\int_{\lambda} a_{\lambda} z^{(\lambda-1)}$ (and we assume henceforth that in (17) all the appropriate limits of (14), (15) and (16) have been taken). We assert that

$$
S \leq \sum_{k, p, s}\left|b_{k p s}\right| \prod_{h q t<k p s}\left(\sigma-1+\left|\alpha_{h}+t-1\right|\right) .
$$

To see this observe that if all quantities appearing are real and positive then already $S=P(\sigma-1)$ and this becomes our assertion (22). Further, $a_{\lambda}$ is given by

$$
(\lambda-1) ! a_{\lambda}=\left\{\left(\frac{\delta}{\delta z}\right)^{\lambda-1} P(z)\right\}_{z=0}
$$


It follows from (2l) that for $1 \leq \lambda \leq \sigma$,

$$
(\lambda-1) !\left|a_{\lambda}\right| \leq\left\{\left(\frac{\delta}{\delta z}\right)^{\lambda-1} \sum_{k, p, s} b_{k p s} \prod_{h q t<k p s}\left(z+\left|\alpha_{h}+t-1\right|\right)\right\}_{z=0},
$$

since

$$
\frac{\delta}{\delta z^{n}} z^{n}=\sum_{\mu=1}^{n}\left(\begin{array}{l}
n \\
\mu
\end{array}\right) z^{n-\mu}
$$

the point being that no negative terms arise. The collection of these remarks amount to our assertion.

\section{9.}

The results (20) and (22) give a precise lower bound for the Theorem. So as to provide a numerical, if cruder, result, write

$$
A=\max _{h, q, t}\left|\alpha_{h}+t-1\right|, 1 \leq h \leq m ; 1 \leq q \leq n(h) ; 1 \leq t \leq \rho(h q) .
$$

Choosing $R=A+1$ in (20) we certainly have

$$
\left|b_{k p s}\right|<\left\{\begin{array}{ll}
(A+1)|1-u|^{-A-1} & \text { if }|1-u|<1 \\
(A+1)|1-u|^{A+1} & \text { if }|1-u| \geq 1
\end{array} .\right.
$$

Hence

(23)

$$
\begin{aligned}
S & <\sum_{i=1}^{\sigma}(A+1)|1-u|^{ \pm(A+1)}(\sigma-1+A)^{\tau-1} \\
& <|1-u|^{ \pm(A+1)}(\sigma+A)^{\sigma} .
\end{aligned}
$$

This result together with the Main Lemma immediately gives the Theorem. We should remark that our estimates are exceptionally crude; a more accurate result appropriate to the particular case can easily be recovered however from the results (20) and (22) above.

\section{References}

[1] A. Baker, "A note on the Padé table", Nederl. Akad. Wetensch. Proc. Ser. A 69 (1966), 596-601. 
[2] S. Dancs and P. Turán, "On the distribution of values of a class of entire functions I, II", PubZ. Math. Debrecen 11 (1964), $257-272$.

[3] A.0. Gelfond, Transcendental and algebraic numbers (Dover Publications, New York, 1960).

[4] Kurt Mahler, "Ein Beweis des Thue-Siegelschen Satzes über die Approximation algebraischer Zahlen für binomische Gleichungen", Math. Ann. 105 (1931), 267-276.

[5] K. Mahler, "On the approximation of logarithms of algebraic numbers", Philos. Trans. Roy. Soc. London Ser. A 245 (1953), 371-398.

[6] K. Mahler, "On a class of entire functions", Acta Math. Acad. Sci. Hungar. 18 (1967), 83-96.

[7] N.-E. Nörlund, Leçons sur les séries d'interpolation (GauthierVillars, Paris, 1926).

[8] Paul Turán, Eine neue Methode in der Analysis und deren Anwendungen (Akadémiai Kiadó, Budapest, 1953).

[9] A.J. van der Poorten, "Generalisations of Turán's main theorems on lower bounds for sums of powers", Bull. Austral. Math. Soc. 2 (1970), 15-37.

[10] A.J. van der Poorten, "On sums of exponential functions I", (to appear).

[11] A.J. van der Poorten, "On sums of exponential functions II", (to appear).

University of New South Wales, Kensington, New South Wales. 